Supporting Online Material for

\title{
A Bioreducible Phosphonoamidate Pro-drug Inhibitor of Enolase: A Proof of Concept Study
}

\author{
Victoria C. Yan, Kristine L. Yang, Elliot S. Ballato, Sunada Khadka, Prakriti Shrestha, \\ Kenisha Arthur, Dimitra K. Georgiou, Mykia Washington, Theresa Tran, Anton H. Poral, Cong- \\ Dat Pham, and Matthew J. Yan, and Florian L. Muller* \\ *aettius@aol.com \\ Department of Cancer Systems Imaging, University of Texas MD Anderson Cancer Center \\ Houston, Texas, 77054, United States
}

\section{Table of Contents}

$\begin{array}{ll}\text { 1. General information } & S 2\end{array}$

$\begin{array}{ll}\text { 2. Synthetic procedures } & S 3\end{array}$

$\begin{array}{lc}\text { 3. Cell culture experiments } & S 5\end{array}$

$\begin{array}{ll}\text { 4. References } & S 8\end{array}$ 


\section{General Information}

General synthetic procedures. All solvents and reagents were purchased from Sigma-Aldrich at the highest commercially available purity and were used without further purification. ${ }^{1} \mathrm{H},{ }^{13} \mathrm{C}$, and ${ }^{31} \mathrm{P}$, NMR were recorded on a Bruker Avance $500 \mathrm{MHz}$ or $300 \mathrm{MHz}$ spectrometer, as indicated. BnHEX was initially synthesized according to previously published procedures ${ }^{1}$; subsequent syntheses were contracted to WuXi AppTec, Shanghai, China. Final compounds were purified via HPLC (Phenomenex Luna-C18. 0-3 min 10\% Buffer B, 21-24 min 100\% Buffer B, 27-30 min, $10 \%$ Buffer B; Buffer $A=\mathrm{H}_{2} \mathrm{O}+0.1 \%$ TFA, Buffer $\mathrm{B}=\mathrm{MeCN}+0.01 \%$ TFA). See Figure $1 \mathrm{~b}$ for a representative synthesis scheme.

General cell culture procedure. Cell culture experiments were performed using the D423-MG cell line. The D423-MG cell lines is 1p36 homozygous deleted from CAMTA1 to SLC25A33; this includes $E N O 1$. Isogenic $E N O 1$ ectopically rescued lines were described previously (pCMV ENO1 5X) $)^{2}$. An ENO1-intact cell line (LN319) was used as a control for sensitivity to Enolase inhibitors. Cells were regularly cultured in Dulbecco's modified Eagle's medium supplemented with $10 \%$ fetal bovine serum.

Abbreviations used: DCM — dichloromethane, DIAD — diisopropyl azodicarboxylate, BnHEX hydrogen (1-(benzyloxy)-2-oxopiperidin-3-yl)phosphonate, TFA-trifluoroacetic acid, THFtetrahydrofuran, $\mathrm{MeOH}$ - methanol, $\mathrm{MeCN}$ - acetonitrile. 


\section{Synthetic procedures}

\section{N-benzyl-P-(1-(benzyloxy)-2-oxopiperidin-3-}

yl)phosphonamidic acid (1). Intermediate (1) was prepared according to previously published procedures ${ }^{3}$. To a solution of triphenylphosphine $(275.87 \mathrm{mg}, 1.06 \mathrm{mmol})$ in DCM $(15 \mathrm{~mL})$, DIAD $(204.50 \mu \mathrm{L}, 1.06 \mathrm{mmol})$ were added with stirring at $0^{\circ} \mathrm{C}$

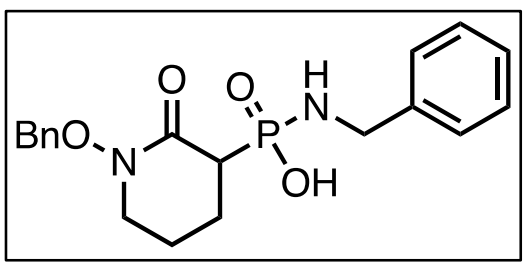
for $30 \mathrm{~min}$. Separately, a solution containing BnHEX (150 mg, $527.74 \mathrm{mmol}$ ), benzylamine $(115.18 \mu \mathrm{L}, 1.06 \mathrm{mmol})$, and DBU $(157.54 \mu \mathrm{L}, 1.06 \mathrm{mmol})$ in DCM (2 mL) was prepared and added dropwise to the betaine solution. The reaction mixture was allowed to warm to room temperature over 30 minutes. Then, 1 volume of water was added to the crude mixture. After vigorous shaking and partitioning via centrifugation $\left(2 \mathrm{~min}\right.$ at $\left.4^{\circ} \mathrm{C}, 4000 \mathrm{rpm}\right)$, the aqueous layer was isolated and lyophilized to a clear solid. Analysis by ESI+ $\left(\mathrm{C}_{19} \mathrm{H}_{23} \mathrm{~N}_{2} \mathrm{O}_{4} \mathrm{P}\right.$ Expected $[\mathrm{M}+\mathrm{H}]^{+}=375.38$. Observed $\left.[\mathrm{M}+\mathrm{H}]^{+}=375.35\right) .{ }^{1} \mathbf{H}$ NMR $\left(500 \mathrm{MHz}, \mathrm{D}_{2} \mathrm{O}\right) \delta 7.32-7.53(\mathrm{~m}, 10 \mathrm{H})$, 4.91-4.96 (m, 2H), 4.02-4.11 (m, 2H), 3.46-3.57 (m, 2H), 2.80-2.87 (dt, J=21.52 Hz, J=21.70 Hz, $1 \mathrm{H}), 2.07-2.13(\mathrm{~m}, 1 \mathrm{H}), 1.92-1.98(\mathrm{~m}, 2 \mathrm{H}), 1.72-1.79(\mathrm{~m}, 1 \mathrm{H}) .{ }^{13} \mathbf{C}$ NMR $\left(125.7 \mathrm{MHz}, \mathrm{D}_{2} \mathrm{O}\right) \delta$ 167.97 (d, J=4.72 Hz, 1C), 141.25 (d, J=7.36 Hz, 1C), 134.52 (s, 1C), 129.92 (s, 2C), 129.15 (s, 1C), 128.73 (s, 2C), 128.60 (s, 2C), 127.59 (s, 1C), 127.00 (s, 1C), 75.54 (s, 1C), 50.11 (s, 1C), 45.28 (s, 1C), 43.64 (d, J=111.12 Hz, 1C), 22.33 (d, J=3.65 Hz, 1C), 21.65 (d, J=7.23 Hz, 1C). ${ }^{31} \mathbf{P}$ NMR $\left(202 \mathrm{MHz}, \mathrm{D}_{2} \mathrm{O}\right) \delta 20.91$.

\section{(((benzylamino)(1-(benzyloxy)-2-oxopiperidin-3-}

yl)phosphoryl)oxy)methyl pivalate (2). To a solution of (1) (36.50 $\mathrm{mg}, 97.24 \mu \mathrm{mol})$ in anhydrous $\mathrm{MeCN}(1 \mathrm{~mL})$, chloromethyl pivalate $(21.02 \mu \mathrm{L}, 145.86 \mu \mathrm{mol})$ and DIPEA $(2 \mu \mathrm{L}, 11.51 \mu \mathrm{mol})$ were added. The reaction was allowed to stir for $15 \mathrm{~h}$ at $50^{\circ} \mathrm{C}$. Then, the crude mixture was concentrated under reduced pressure to a yellow

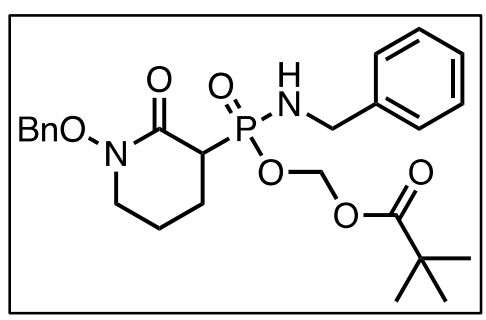
oil and purified via reverse-phase HPLC (Agilent G1361A 1260 Infinity) using a stepwise gradient (5-90\% Buffer B over 10 minutes, 90-100\% Buffer B over 7 minutes, 100\% Buffer B over 8 minutes, $100-5 \%$ Buffer B over 5 minutes; Buffer A: $\mathrm{dH}_{2} \mathrm{O}$ with $0.1 \%$ TFA, Buffer B: $\mathrm{CH}_{3} \mathrm{CN}+$ $0.1 \%$ TFA). The desired compound was obtained after 10 minutes and lyophilized to a white powder. Analysis by ESI+ $\left(\mathrm{C}_{25} \mathrm{H}_{33} \mathrm{~N}_{2} \mathrm{O}_{6} \mathrm{P}\right.$ Expected $[\mathrm{M}+\mathrm{H}]^{+}=490.51$. Observed $\left.[\mathrm{M}+\mathrm{H}]^{+}=490.41\right)$. ${ }^{1} \mathbf{H}$ NMR $\left(500 \mathrm{MHz}, \mathrm{CDCl}_{3}\right) \delta 8.81(\mathrm{~d}, \mathrm{~J}=4.77 \mathrm{~Hz}, 1 \mathrm{H}), 8.24-8.27(\mathrm{t}, \mathrm{J}=7.80,7.87 \mathrm{~Hz}, 1 \mathrm{H}), 7.90$ $7.92(\mathrm{~d}, \mathrm{~J}=7.99 \mathrm{~Hz}, 1 \mathrm{H}), 7.69-7.71(\mathrm{t}, \mathrm{J}=6.44,6.75 \mathrm{~Hz}, 1 \mathrm{H}), 7.33-7.40(\mathrm{~m}, 5 \mathrm{H}), 5.61-5.68(\mathrm{~m}, 2 \mathrm{H})$, $4.90(\mathrm{~d}, \mathrm{~J}=2.22 \mathrm{~Hz}, 2 \mathrm{H}), 4.64-4.80(\mathrm{t}, \mathrm{J}=9.91,9.91 \mathrm{~Hz}, 2 \mathrm{H}), 3.35-3.37(\mathrm{~m}, 2 \mathrm{H}), 3.12-3.20$ (dt, $\mathrm{J}=23.65 \mathrm{~Hz}, 1 \mathrm{H}), 2.09-2.15(\mathrm{~m}, 1 \mathrm{H}), 1.94-2.05(\mathrm{~m}, 2 \mathrm{H}), 1.69-1.75(\mathrm{~m}, 1 \mathrm{H}), 1.18(\mathrm{~s}, 9 \mathrm{H}) .{ }^{13} \mathrm{C} \mathrm{NMR}$ $\left(125 \mathrm{MHz}, \mathrm{CDCl}_{3}\right) \delta 177.48(\mathrm{~d}, \mathrm{~J}=7.39 \mathrm{~Hz}, 1 \mathrm{C}), 163.53$ (d, J=4.81 Hz, 1C), 156.93 (d, J=4.22 Hz, 1C), 145.22 (s, 1C), 143.48 (s, 1C), 130.13 (s, 2C), 130.03 (s, 1C), 129.42 (s, 1C), 129.03 (s, 2C), 126.04 (s, 1C), 125.18 (s, 1C), 81.58-81.64 (d, J=7.40 Hz, 1C), 76.40 (s, 1C), 43.73-44.77 (d, 
$\mathrm{J}=132.02 \mathrm{~Hz}, 1 \mathrm{C}), 43.05$ (s, 1C), 22.43-22.47 (d, J=4.61 Hz, 1C), 21.91-21.94 (d, J=4.32 Hz, 1C); quaternary $\mathrm{sp}^{3}$ carbon not observed. ${ }^{31} \mathbf{P}$ NMR $\left(202 \mathrm{MHz}, \mathrm{CDCl}_{3}\right) \delta 29.81(\mathrm{~s}, 1 \mathrm{P}), 29.11(\mathrm{~s}, 1 \mathrm{P})$ (isomers).

\section{(((Benzylamino)(1-hydroxy-2-oxopiperidin-3-} yl)phosphoryl)oxy)methyl pivalate (3, FLM37). Palladium on carbon $(10$ wt. $\%, 100 \mathrm{mg})$ was mixed with THF $(2 \mathrm{~mL})$ and $\mathrm{MeOH}(3 \mathrm{~mL})$ with stirring. Hydrogen in a balloon was then allowed to flow through the reaction for 1 minute with venting. Then, a second balloon with

hydrogen was added to the reaction and the mixture was allowed to stir

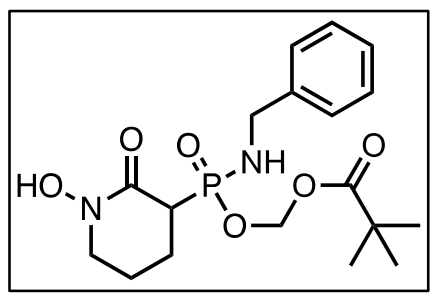
and saturate with hydrogen for 1 hour. Next, to a separate vial, (2) was dissolved in $\mathrm{MeOH}$ (2 mL) and injected into the hydrogen-charged flask. The reaction stirred at room temperature for 1 hour. Then, the palladium was filtered, and the reaction was concentrated under reduced pressure to a pale-yellow oil. The crude product was purified via reverse-phase HPLC (Agilent G1361A 1260 Infinity) using a stepwise gradient (1-60\% Buffer B over 25 minutes, 60-100\% Buffer B over 10 minutes, 100\% Buffer B over 5 minutes, 100-0\% Buffer B over 1 minutes; Buffer A: $\mathrm{dH}_{2} \mathrm{O}$ with $0.1 \%$ TFA, Buffer B: $\mathrm{CH}_{3} \mathrm{CN}+0.1 \%$ TFA). Product-containing fractions were combined and lyophilized to a pale-yellow solid. Analysis by ESI+ $\left(\mathrm{C}_{18} \mathrm{H}_{27} \mathrm{~N}_{2} \mathrm{O}_{6} \mathrm{P}\right.$ Expected $[\mathrm{M}+\mathrm{H}]^{+}=399.40$. Observed $\left.[\mathrm{M}+\mathrm{H}]^{+}=399.35\right) .{ }^{1} \mathbf{H}$ NMR $\left(500 \mathrm{MHz}, \mathrm{CDCl}_{3}\right) \delta$ 7.22-7.39 (m, 5H), 5.59-5.66 (dd, $\mathrm{J}=12.32 \mathrm{~Hz}, 2 \mathrm{H}), 5.54-5.59(\mathrm{ds}, \mathrm{J}=12.66 \mathrm{~Hz}, 2 \mathrm{H}), 4.29-436(\mathrm{t}, \mathrm{J}=11.71 \mathrm{~Hz}, 2 \mathrm{H}), 4.25-4.32(\mathrm{t}$, $\mathrm{J}=11.29 \mathrm{~Hz}, 2 \mathrm{H}), 3.01-3.10(\mathrm{dt}, \mathrm{J}=22.99 \mathrm{~Hz}, 1 \mathrm{H}), 2.86-2.95(\mathrm{dt}, \mathrm{J}=22.79 \mathrm{~Hz}, 1 \mathrm{H}), 2.08-2.13(\mathrm{~m}, 2 \mathrm{H})$, 1.83-2.08 (m, 2H), 1.20 (s, 9H), $1.19(\mathrm{~s}, 9 \mathrm{H}) .{ }^{13} \mathbf{C ~ N M R}\left(125.7 \mathrm{MHz}, \mathrm{CDCl}_{3}\right) \delta 177.26(\mathrm{~s}, 1 \mathrm{C})$, 177.23 (s, 1C), 160.33-160.43 (d, 1.29Hz, 1C), 160.17-160.31 (d, J=2.96Hz, 1C), 139.94 (d, $\mathrm{J}=2.72 \mathrm{~Hz}, 1 \mathrm{C}), 139.4-139.52(\mathrm{~d}, 2.53 \mathrm{~Hz}, 1 \mathrm{C}), 127.27-1128.70(\mathrm{~m}, 5 \mathrm{C}), 81.26-81.45(\mathrm{~d}, \mathrm{~J}-3.11 \mathrm{~Hz}$, 1C), 80.87-81.05 (d, J=3.60Hz, 1C), 48.67 (s, 1C), 40.89-41.21 (d, J=31.86Hz, 1C), 26.81-26.90 (s, 3C), 21.43-21.85 (m, 2C). ${ }^{31} \mathbf{P}$ NMR (202 MHz, $\left.\mathrm{CDCl}_{3}\right) \delta 28.76$ (s, 1P), 29.63 (s, 1P) (isomers).

\section{N-benzyl-P-(1-hydroxy-2-oxopiperidin-3-yl)phosphonamidic} acid (4). A solution of $10 \% \mathrm{Pd} / \mathrm{C}(200 \mathrm{mg})$ in anhydrous THF/MeOH (2:3) was stirred at $25^{\circ} \mathrm{C}$. A balloon of $\mathrm{H}_{2}$ was added and the solution vented for 10 minutes. A second balloon of $\mathrm{H}_{2}$ was then added, and the solution stirred for $1 \mathrm{~h}$. Then, this slurry was

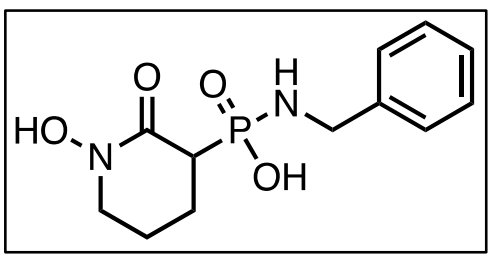
transferred to a vial containing BnFLM38 $(250 \mathrm{mg}, 668 \mathrm{mmol})$ and was allowed to stir for $1 \mathrm{~h}$. The reaction was filtered and concentrated to a yellow oil. Analysis by ESI+ $\left(\mathrm{C}_{12} \mathrm{H}_{16} \mathrm{~N}_{2} \mathrm{O}_{6} \mathrm{P}\right.$ Expected $[\mathrm{M}+\mathrm{H}]^{+}=285.25$ Observed $\left.[\mathrm{M}+\mathrm{H}]^{+}=285.30\right)$. 
P-(1-acetoxy-2-oxopiperidin-3-yl)-N-benzylphosphonamidic acid (5). To a solution of (4) (65 mg, $229 \mu \mathrm{mol})$ in anhydrous MeCN $(500 \mathrm{uL}), \mathrm{Ac}_{2} \mathrm{O}(65 \mu \mathrm{L}, 668 \mu \mathrm{mol})$ was added. The reaction stirred for $3 \mathrm{~h}$ at $25^{\circ} \mathrm{C}$. Then, the reaction was concentrated and lyophilized for 2 days. Analysis by ESI+ $\left(\mathrm{C}_{14} \mathrm{H}_{18} \mathrm{~N}_{2} \mathrm{O}_{6} \mathrm{P}\right.$ Expected

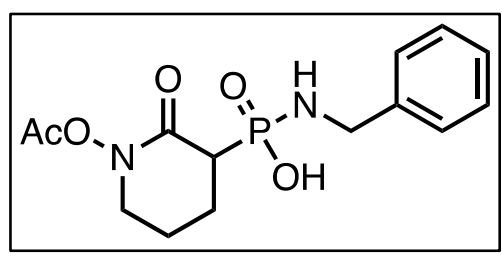
$[\mathrm{M}+\mathrm{H}]^{+}=327.29$ Observed $\left.[\mathrm{M}+\mathrm{H}]^{+}=327.32\right) .{ }^{13} \mathbf{C ~ N M R}\left(75 \mathrm{MHz}, \mathrm{CDCl}_{3}\right) \delta 168.01(\mathrm{~s}, 1 \mathrm{C}), 161.65$ (d, J=6.32 Hz, 1C), 141.92 (d, J=6.96 Hz, 1C), 128.22 (s, 2C), 127.55 (s, 2C), 126.54 (s, 1C), 51.63 (s, 1C), 43.24 (s, 1C), 36.42-35.29 (d, J=170.11 Hz, 1C), 25.48 (s, 1C), 25.14 (5.93 Hz, 1C), 22.13 $(\mathrm{d}, \mathrm{J}=7.06 \mathrm{~Hz}, 1 \mathrm{C}) .{ }^{31} \mathbf{P}$ NMR $\left(121 \mathrm{MHz}, \mathrm{CDCl}_{3}\right) \delta 17.50$ (s, 1P).

\section{3-((benzylamino)((5-nitrofuran-2-yl)methoxy)phosphoryl)-2-} oxopiperidin-1-yl acetate (6, VCY15). To a solution of (5) (73 $\mathrm{mg}, 224 \mathrm{mmol}$ ) in anhydrous acetonitrile, 2-(bromomethyl)-5nitrofuran (138.26 $\mathrm{mg}, 671 \mathrm{mmol}$ ) were added. The reaction was allowed to stir at $50^{\circ} \mathrm{C}$ for $20 \mathrm{~h}$. The solvent was then removed and the reaction was purified via reverse-phase HPLC (1-60\% Buffer B over 25 minutes, 60-100\% Buffer B over 10 minutes, 100\% Buffer

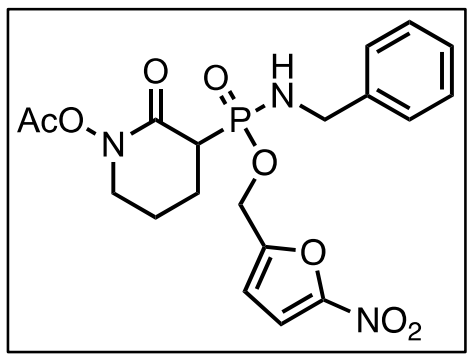
B over 5 minutes, 100-0\% Buffer B over 1 minutes; Buffer $\mathrm{A}$ : $\mathrm{dH}_{2} \mathrm{O}$ with $0.1 \%$ TFA, Buffer B: $\mathrm{CH}_{3} \mathrm{CN}+0.1 \%$ TFA). Product-containing fractions were combined and lyophilized to a yellow solid. Analysis by ESI+ $\left(\mathrm{C}_{19} \mathrm{H}_{22} \mathrm{~N}_{3} \mathrm{O}_{8} \mathrm{P}\right.$ Expected $[\mathrm{M}+\mathrm{H}]^{+}=452.37$. Observed $\left.[\mathrm{M}+\mathrm{H}]^{+}=452.42\right) .{ }^{1} \mathbf{H}$ NMR $\left(300 \mathrm{MHz}, \mathrm{CDCl}_{3}\right)$ 7.26-7.23 (m, 6H), 7.17-7.12 (d, J=3.82 Hz, 1H), 6.49 (d, J=7.12 Hz, 2H), $4.89(\mathrm{~m}, 2 \mathrm{H}), 4.36(\mathrm{~d}, \mathrm{~J}=3.22 \mathrm{~Hz}, 2 \mathrm{H}), 4.22(\mathrm{~d}, \mathrm{~J}=5.92 \mathrm{~Hz}, 2 \mathrm{H}), 3.62(\mathrm{~m}, 2 \mathrm{H}), 3.04$ (dt, $\mathrm{J}=23.40,7.58 \mathrm{~Hz}, 1 \mathrm{H}), 2.13(\mathrm{~s}, 3 \mathrm{H}), 1.96(\mathrm{~s}, 2 \mathrm{H}), 1.18(\mathrm{~s}, 2 \mathrm{H}) .{ }^{13} \mathbf{C} \mathbf{N M R}\left(75 \mathrm{MHz}, \mathrm{CDCl}_{3}\right) \delta$ 170.19 (s, 1C), 167.26 (d, J=15.44 Hz, 1C), 153.35 (d, J=9.55 Hz, 1C), 152.60 (s, 1C), 140.09 (d, $\mathrm{J}=2.07 \mathrm{~Hz}, 1 \mathrm{C}), 128.83-127.24$ (m, 5C), 112.62-111.99 (m, 2C), 57.39 (s, 1C), 51.37 (s, 1C), 44.46 (s, 1C), 43.61 (d, J=62.07 Hz, 1C), 22.52 (d, J=6.32 Hz, 1C), 20.67 (d, J=2.58 Hz, 1C), 18.2 (s, 1C). ${ }^{31}$ P NMR (121 MHz, $\mathrm{CDCl}_{3}$ ) $\delta 29.49$ (s, 1P), 29.37 (s, 1P) (isomers). 


\section{Cell culture experiments.}

Cell viability assay. Cell viability was determined by crystal violet staining, as previously described $^{4,5}$. The cell lines used were D423 (ENO1-deleted), D423 ENO1 (overexpressing ENO1) and LN319 (control). Glioma cells were seeded in 96-well plates and treated with varying concentrations of the inhibitors described above for 7 days. Cells were then washed with PBS, fixed with $10 \%$ formalin and stained with $0.05 \%$ crystal violet. Washed and dried plates were dyeextracted using $10 \%$ acetic acid, and absorbance was measured at $595 \mathrm{~nm}$ using Omegastar Plate Reader (BMG Labtech). To test the efficacy of the synthesized under hypoxic conditions, $1 \mathrm{x}$ $10^{4}$ cells were plated in 96-well plates, treated with inhibitor and incubated for 3 days in a hypoxia station (Don Whitley Scientific, Shipley, UK) set at $1 \% \mathrm{O}_{2}$ and $5 \% \mathrm{CO}_{2}$. Crystal violet staining was then performed as described above.

\section{Representative plates for VCY15, POMHEX, and TH-302}

\section{VCY15}

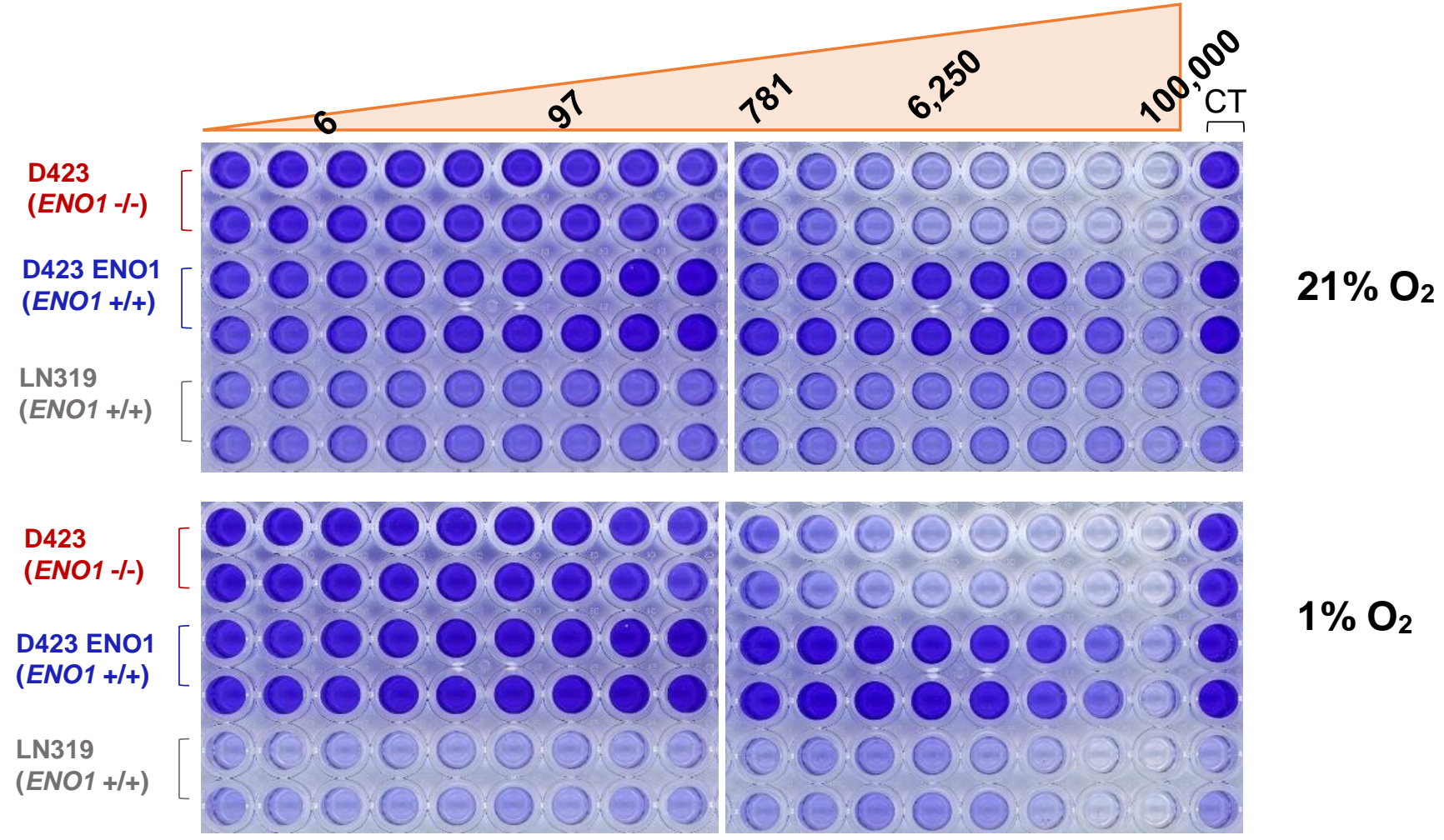




\section{POMHEX}

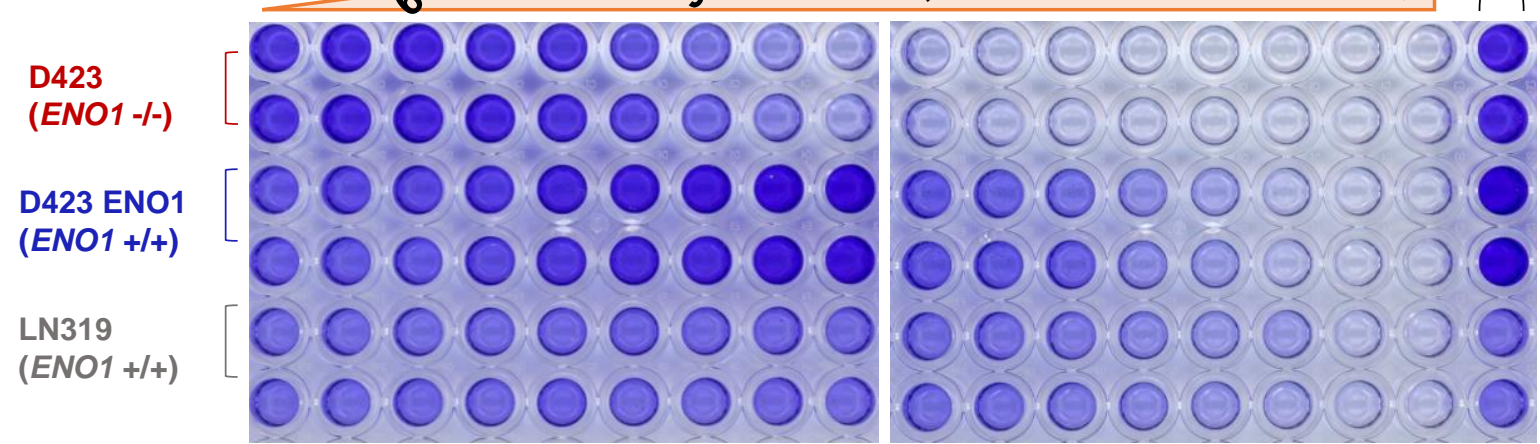

$21 \% \mathrm{O}_{2}$

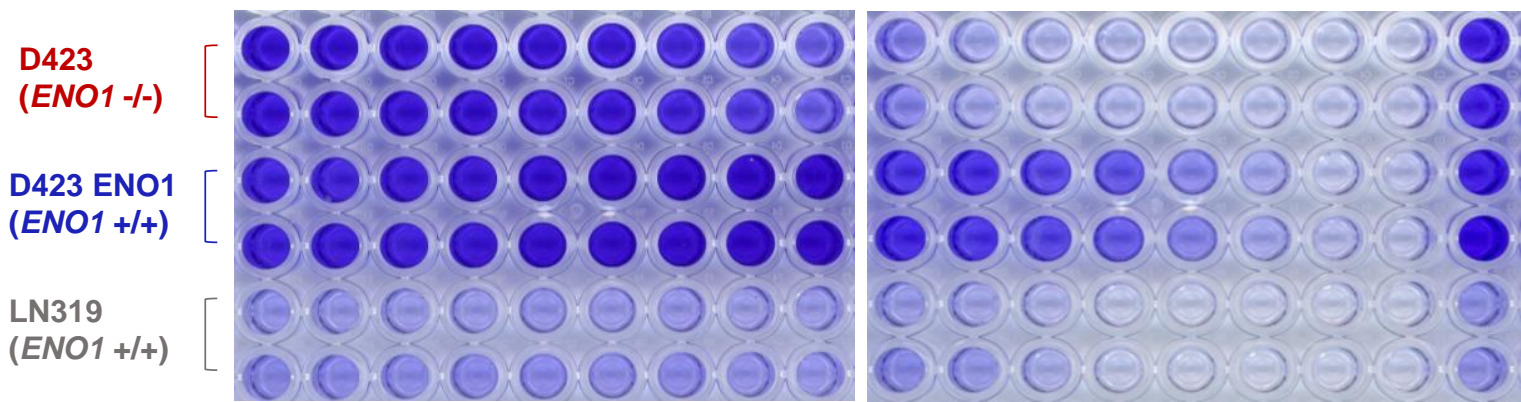

$1 \% \mathrm{O}_{2}$

\section{TH-302}

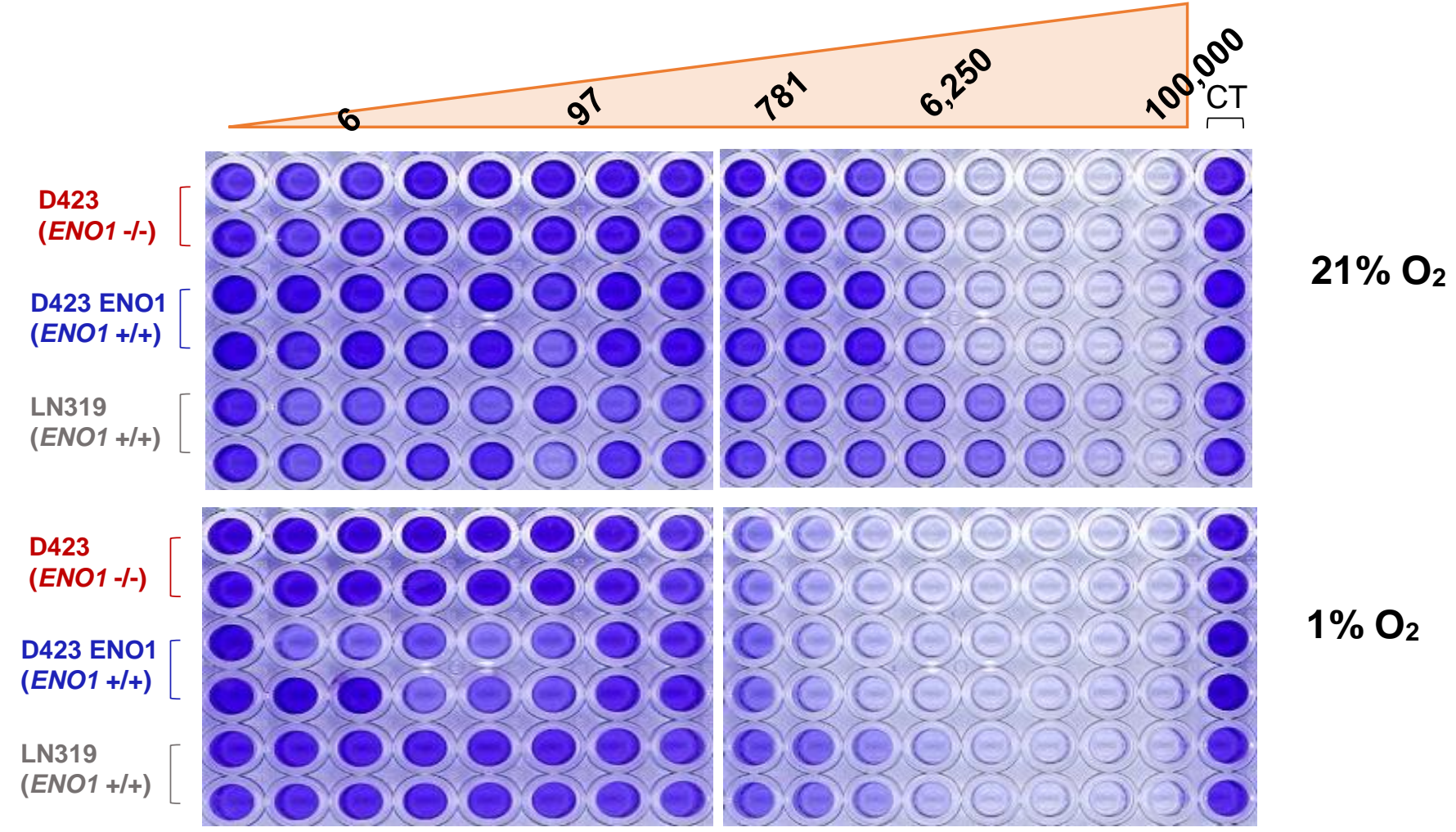




\section{References}

(1) Lin, Y.-H.; Satani, N.; Hammoudi, N.; Ackroyd, J. J.; Khadka, S.; Yan, V. C.; Georgiou, D. K.; Sun, Y.; Zielinski, R.; Tran, T.; et al. Eradication of ENO1-Deleted Glioblastoma through Collateral Lethality. bioRxiv 2018, 331538.

(2) Muller, F. L.; Colla, S.; Aquilanti, E.; Manzo, V. E.; Genovese, G.; Lee, J.; Eisenson, D.; Narurkar, R.; Deng, P.; Nezi, L.; et al. Passenger Deletions Generate Therapeutic Vulnerabilities in Cancer. Nature 2012, 488, 337-343.

(3) Yan, V. C.; Pham, C.-D.; Muller, F. L. Expedient Method for Direct Mono-Amidation of Phosphonic and Phosphoric Acids. ChemRxiv 2020.

(4) Leonard, P. G.; Satani, N.; Maxwell, D.; Lin, Y.-H.; Hammoudi, N.; Peng, Z.; Pisaneschi, F.; Link, T. M.; Lee, G. R.; Sun, D.; et al. SF2312 Is a Natural Phosphonate Inhibitor of Enolase. Nat. Chem. Biol. 2016, 12 (12), 1053-1058.

(5) Satani, N.; Lin, Y.-H.; Hammoudi, N.; Raghavan, S.; Georgiou, D. K.; Muller, F. L. ENOblock Does Not Inhibit the Activity of the Glycolytic Enzyme Enolase. PLoS One 2016, 11 (12), e0168739. 